\title{
sEMG based Classification of Hand Gestures using EMD Algorithm and its Application on Control System
}

\author{
Rahul Bhadra Bajracharya, Sujan Bhusal, S. Jagadeesan
}

\begin{abstract}
Single sensor is employed for classifying four hand gestures from flexor carpum ulnaris. The first three IMFs that are obtained as a result of Empirical Mode Decomposition are taken into consideration. Time domain features like mean, variance, skewness, etc are taken for each IMFs. Support Vector Machine was used for classification task and the extracted model is used for making predictions
\end{abstract}

\section{INTRODUCTION}

Signal processing has been fascinating field of research over few decades as signal carries information. Similarly myoelectric signal contains information about muscle movement. Detecting the hand gesture based on myoelectric signal is what researcher are interested now a days. There are multiple uses of myoelectric control systems some of which includes prosthetic hand control, rehabilitation, human-computer interface and so on. The action potential that causes the hand movements are captured by surface electromyography(sEMG) and the data are classified based on feature vectors that contains useful information which differentiate one class of hand gesture with another.

This paper demonstrates the experimental result of using single channel EMG recordings for classifying the hand gestures and using the classified model to interact with objects in python. In this experiment the EMG signals are acquired using single channel myoware sensor attached on flexor carpum ulnaris. EMD, a advanced signal processing technique which has proved to enhance the classification is being used. After applying EMD algorithm the feature vectors of the relevant IMFs(Intrinsic Mode Function) were extracted and then Support Vector Machine(SVM) was used to classify the hand gesture.

\section{EXPERIMENTAL SETUP}

In total 4 classes of hand gestures namely open ,close, extension and flexion have been studied in our research work. A single channel Myo-ware sensor was used to acquire the EMG signal. 5 persons: 3 male and 2 female of age group

Revised Manuscript Received on September 22, 2019.

Rahul Bhadra Bajracharya, SRM Institute Of Science and Technology. rahulbajra16@gmail.com,

Sujan Bhusal, SRM Institute Of Science and Technology. sujanbhusal1@gmail.com,

S. Jagadeesan, SRM Institute Of Science and Technology. jagadeesan.s@ktr.srmuniv.ac.in
20-30 were involved in collecting the data who have no history of neuromuscular disease. A total of 25 readings for each class of gesture per individual resulted in $100\left(25^{* 4}\right.$ gesture reading per individual)*5(person) which sums to a total of 500 data. Sensor was placed on Flexor carpum ulnaris muscle.

\section{Empirical Mode Decomposition:}

Since the EMG signals are considered non-stationary signals EMD algorithm is used to decompose the original signals to IMFs and only relevant IMFs are selected for feature extraction.IMF is defined as a function that satisfies the following requirements:

- "The number of extrema and the number of zero-crossing must be equal or differ at most by one."(i.e signal is oscillatory in nature)

- "At any point the mean value of envelope defined by local maxima and local minima is zero."(i.e frequency can vary over period of time)

\section{AlgorithmforEMD}

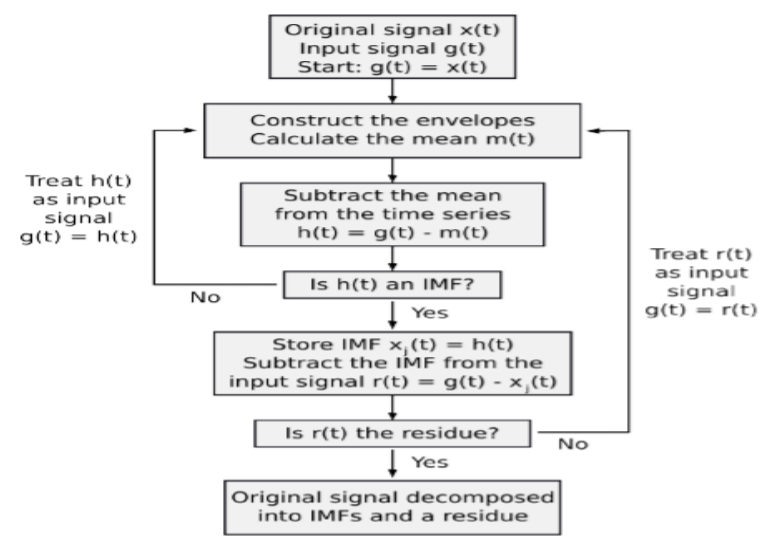

Important step after applying EMD algorithm is the selection of relevant IMFs. Peng et al(2005) purposed an expression to determine relevant IMFs. The concept is based on the fact that irrelevant IMFs will have low correlation with the original signal than the relevant ones. Therefore a threshold is introduced by

$\lambda=\frac{\max \left(\mu_{i}\right)}{10}, i=1,2 \ldots . n$ 
Applying this equation we found first three IMFs contained enough relevant information about the signal.

\section{Hilbert-Spectrum:}

The Hilbert spectrum is a statistical tool that distinguishes among the mixture of moving signals. The spectrum is decomposed into component sources.It is a 2-step process:

- Preprocessing a signal into intrinsic mode function using decomposition algorithm like empirical mode decomposition(EMD)

- .Applying the Hilbert transform to get instantaneous frequency spectrum of eachcomponents and also the instantaneous energy.

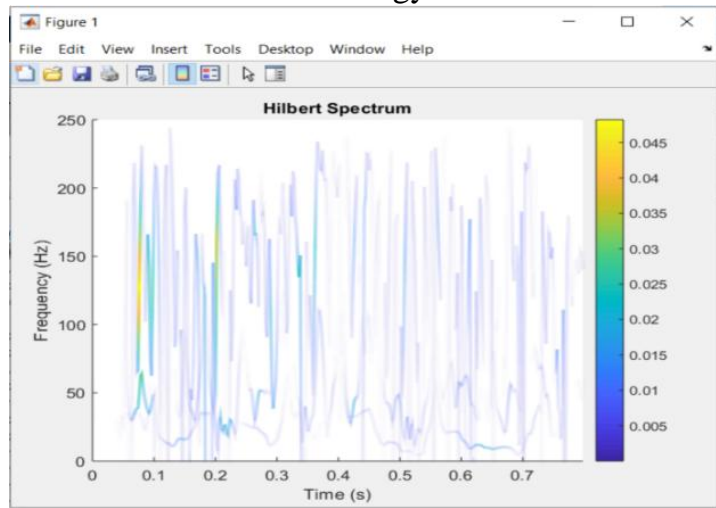

Fig:Hilbert Spectrum of gesture:open

\section{Feature Extraction and Classification:}

As raw signals are rarely involved in pattern recognition, a feature extraction process is carried out to extract the relevant information so that those information can be used to classify the model. In our project simple features like mean ,
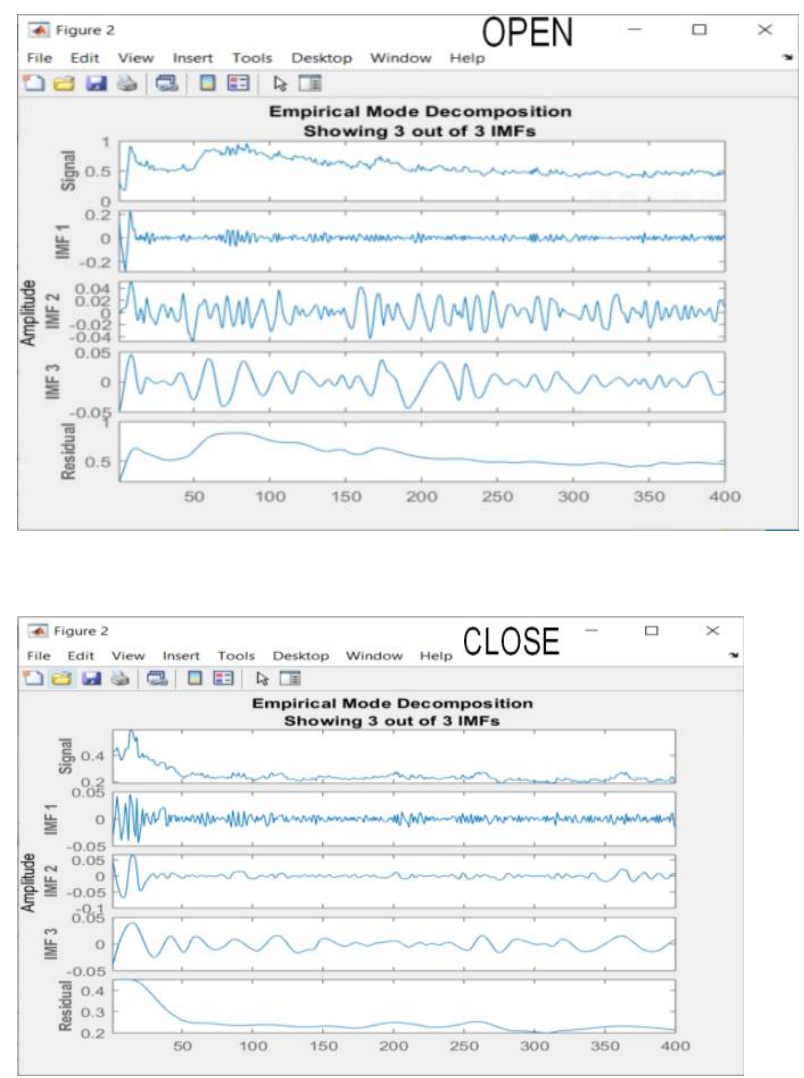
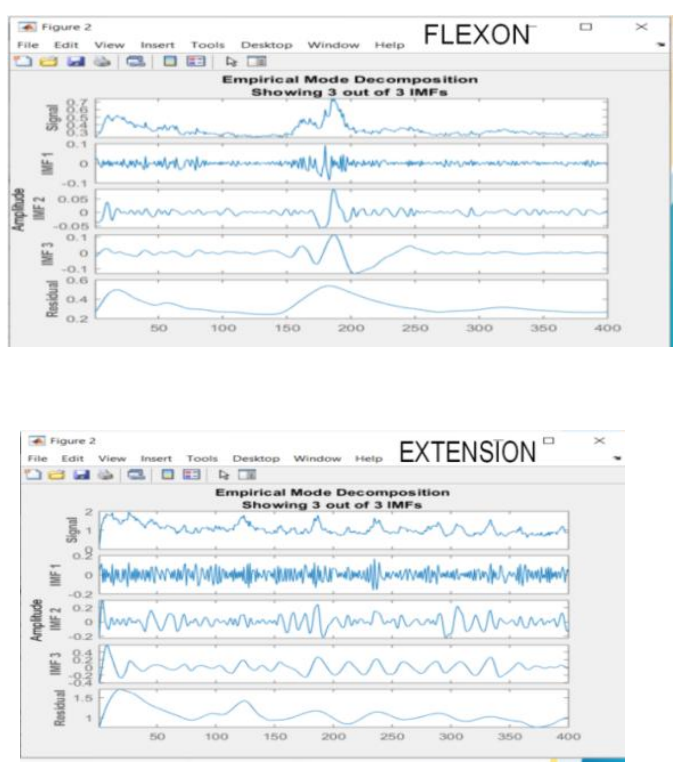

variance,skewness, and standard deviation of each imf, mean of instantaneous energy of each imf and mean of instantaneous frequency of each imf were used. Using this feature vectors different classification algorithm were tested upon among which Support Vector Machine(SVM) seemed to be promising. Since only one channel sEMG electrode were used accuracy for 4 classes of gesture namely open,close, extension and flexion reached around $73 \%$ and when flexion class was removed and model was trained for 3 classes of gesture accuracy reached around 92\%. Looking at the confusion matrix of the former we can see close and flexion were not easily separable through which we can conclude the selected muscle was less appropriate to distinguish between these two classes or that the electrodes numbers were insufficient and the classification accuracy can be improved by increasing the channel of electrodes or can be improved by selecting any other muscles.

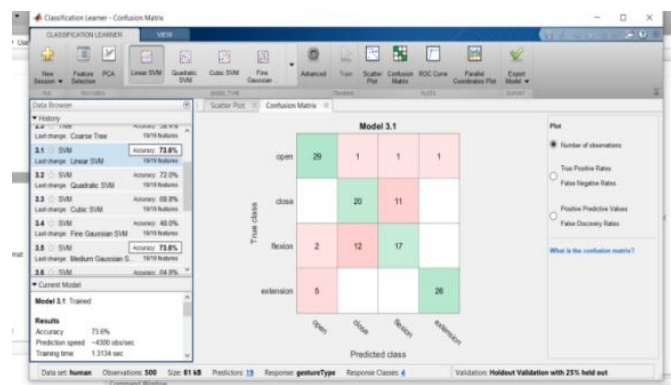

Fig: Confusion matrix for 4 classes of gestures

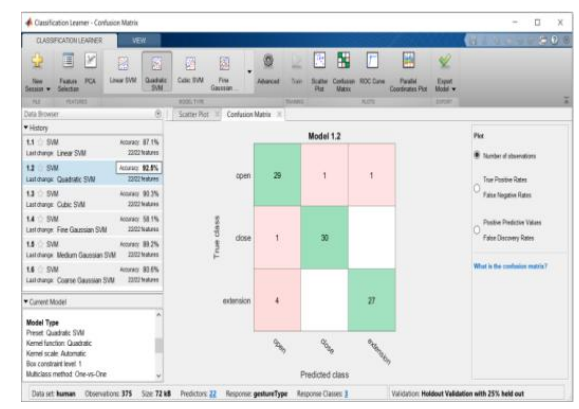

Fig: Confusion matrix for 3 classes of gestures

Published By:

Blue Eyes Intelligence Engineering 


\section{Output:}

After the model was trained the model was used for making prediction on new real time data and based on the prediction made the control system was made that was responsible for the movement of object in python environment. The python environment simply contained the code for movement of the object and the respective module was called from matlab based on what the predicted class of gesture was(i.e extension gesture resulted in calling the python module that contained code for movement of object in $\mathrm{x}$-axis and so on).
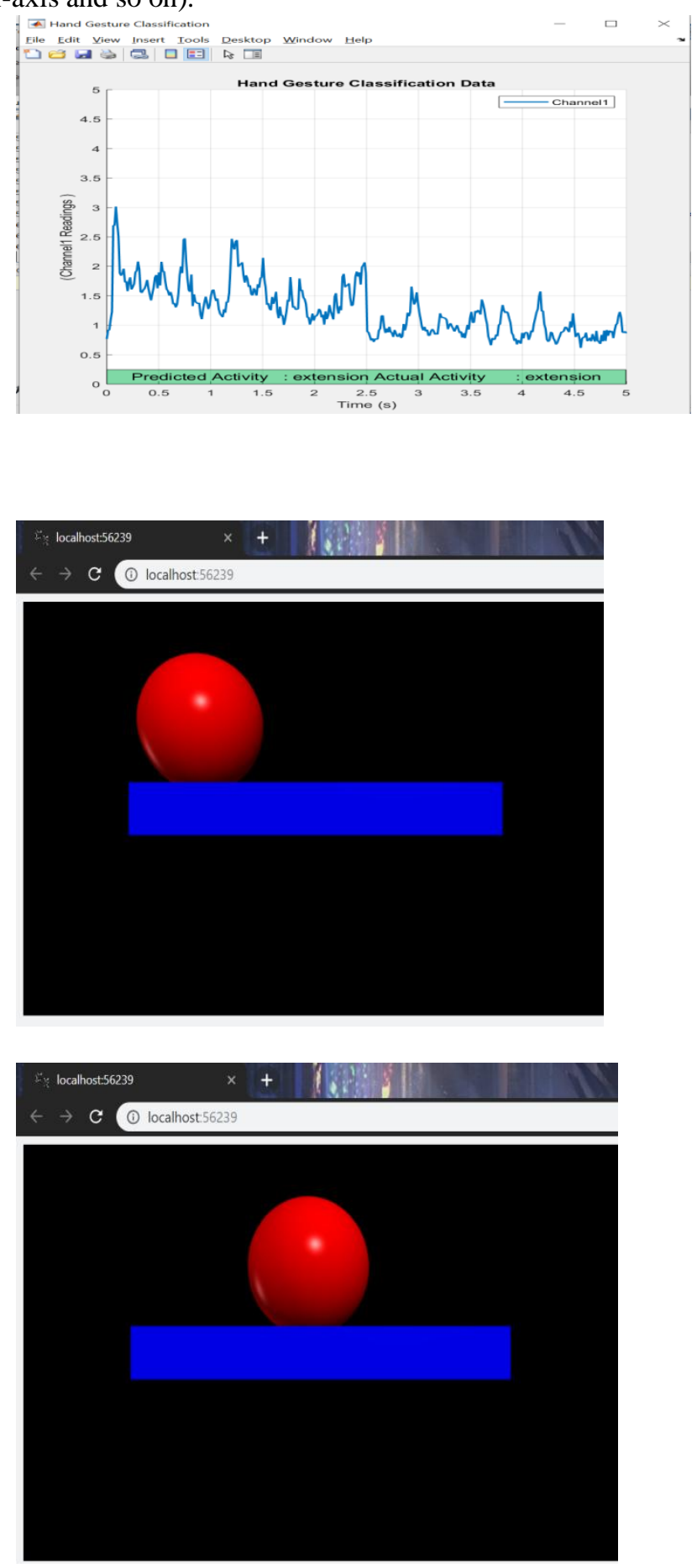

\section{RESULT \& FUTURE ENHANCEMENT:}

Muscle activity onset detection was not performed in our project that led to collection of data before the onset which were insignificant leading to low accuracy of classification. If muscle activity onset would have been identified the data that were collected after onset only would have been used to extract the feature and the accuracy would have increased. The new technique and the promising one TKEO will be used to detect the onset time.Threshold-based algorithm can be applied to TKEO output to locate onset time of muscle activityXiaoyan et al (2006) .

$$
D F=u_{o}+j . o_{o}
$$

where $u_{o}$ is mean and $o_{o}$ is standard deviation.

The preset variable $\mathrm{j}$ can be identified empirically.

\section{ACKNOWLEDGMENT}

We would like to thank S.Jagadeesan our guide who helped us in every step of the project

\section{REFERENCES}

1) Chen, X., Zhang, X., Zhao, Z. Y., Yang, J. H., Lantz, V., and Wang, K. Q. (2007a). "Hand gesture recognition research based on surface EMG sensors and 2D-accelerometers." Proceedings - International Symposium on Wearable Computers, ISWC,11-14.

2) Chen, X., Zhang, X., Zhao, Z. Y., Yang, J. H., Lantz, V., and Wang, K. Q. (2007b). "Multiple hand gesture recognition based on surface EMG signal." 2007 1st Interna- tional Conference on Bioinformatics and Biomedical Engineering, ICBBE,506-509.

3) Coogan, C. G. and He, B. (2018). "Brain-Computer Interface Control in a Virtual Reality Environment and Applications for the Internet of Things." IEEE Access, 6(c), 10840-10849.

4) Edith, R. M. and Bhargavi Haripriya, A. (2015). "Gesture recognition using real time EMG." ICIIECS 2015 - 2015 IEEE International Conference on Innovations in Infor- mation, Embedded and Communication Systems,2-4.

5) 5.G., Y., J., D., G., P., H., Z., J., L., B., D., H., X., H., Y., Z., P., J., X., M., J., and P., L.(2018).“AnIoT-Enabled Stroke Rehabilitation System Based on Smart WearableArmband andMachineLearning."IEEEJournalofTranslationalEngineeringinHeal th and Medicine,6(May).

6) KarlÄs'k, B. (2014). "Machine Learning Algorithms for Characterization of EMG Sig- nals." International Journal of Information and Electronics Engineering,4(3).

7) Nagata,K.,Adno,K.,Magatani,K.,andYamada,M.(2006).“AClassificati onMethod of Hand Movements Using Multi Channel Electrode."2375-2378.

8) Peng, Z. K., Tse Peter, W. and Chu, F. L. (2005). A comparison study of improved Hilbert-Huang transform and wavelet transform: Application to fault diagnosis for rolling bearing. Mech. Syst. Signal Process., 19: 974-988.

9) Sapsanis, C., Georgoulas, G., and Tzes, A. (2013). "EMG based classification of basic hand movements based on time frequency features." $2013 \quad 21 \mathrm{st} \quad$ MediterraneanConferenceonControlandAutomation,MED2013-ConferenceProceedings,(M arch2016), 716-722.

10) Tomizawa, K., Watanabe, K., Kobayashi, K., and Kurihara, Y. (2011) "Detection of Surface Myoelectric Signals Using a Small Number of Electrodes Contraction Power spectrumFrequencys $(\mathrm{Hz})$ ExtensionPowerspectrumFrequencies(Hz).”2 313-2316.

11) Wheeler,K.R.andCharles,C.(2003).“"gesturesasInput:InputMethodsfor Wearable Devices."

12) Xiaoyan Li and Aruin, A. (2006). "Muscle Activity Onset Time Detection Using Teager-Kaiser Energy Operator.”7549-7552. 